\title{
Dickkopf proteins influence lung epithelial cell proliferation in idiopathic pulmonary
} fibrosis

\author{
E-M. Pfaff*, S. Becker*, A. Günther* and M. Königshoff ${ }^{\#, \uparrow}$
}

ABSTRACT: Idiopathic pulmonary fibrosis (IPF) is a fatal interstitial lung disease with unknown pathogenesis. The WNT/ $\beta$-catenin pathway has recently been reported to be operative in epithelial cells in IPF. Dickkopf (DKK) proteins are known to regulate WNT signalling via interaction with Kremen (KRM) receptors, yet their expression and role in the adult lung and in IPF has not been addressed.

We analysed the expression, localisation and function of DKK and KRM proteins in IPF lungs using Western blotting, quantitative RT-PCR, immunohistochemistry, ELISA and functional in vitro studies.

Enhanced expression of DKK1 and DKK4 and KRM1 was detected in lung homogenates of IPF patients compared with transplant donors. Immunohistochemistry revealed that DKK1 was predominantly localised in basal bronchial epithelial cells. Furthermore, prominent expression of all proteins was observed in hyperplastic alveolar epithelial cells in IPF. Quantitative measurement of DKK1 revealed enhanced protein expression in the bronchoalveolar lumen of IPF patients. Finally, functional studies using human bronchial and alveolar epithelial cell lines demonstrated that WNT-induced epithelial cell proliferation is regulated by DKK1 in a dosedependent fashion.

In summary, DKK proteins are expressed in the lung epithelium in IPF. DKK proteins influence epithelial cell proliferation and may, therefore, be suitable therapeutic targets for IPF.

KEYWORDS: Dickkopf proteins, lung epithelial cells, pulmonary fibrosis, WNT signalling

diopathic pulmonary fibrosis (IPF) is a progressive and fatal interstitial lung disease with unknown pathogenesis and limited responsiveness to current therapies [1-3]. It is the most common form of idiopathic interstitial pneumonias, which are characterised by destruction of lung architecture and loss of respiratory function $[1,4,5]$. The histological pattern of IPF is usual interstitial pneumonia (UIP) [6, 7], and aggregates of activated myofibroblasts, so-called fibroblast foci, are hallmark lesions of IPF/UIP. It has been proposed that repetitive alveolar injury leads to initial alveolar epithelial cell death, subsequent hyperplasia and aberrant activation of the alveolar epithelium $[8,9]$. The subepithelial localisation of fibroblast foci in these areas suggest that impaired epithelial-mesenchymal crosstalk contributes to the pathobiology of IPF $[8,10,11]$.

The WNT family of proteins, highly conserved secreted growth factors are known to control key events during lung development $[12,13]$. WNT signalling is regulated via binding of extracellular
WNT ligands to receptors of the frizzled family or low density lipoprotein receptor-related proteins (LRP). The best characterised WNT signalling pathway is the $\beta$-catenin-dependent, or canonical, WNT signalling pathway. In unstimulated cells, $\beta$-catenin, the main signalling intermediate of canonical WNT signalling, is bound to the scaffold proteins axin and adenomatosis polyposis coli, and constitutively phosphorylated by its interaction with casein kinase I and glycogen synthase kinase-3 $\beta$ and degraded. Upon WNT stimulation, the LRP6 receptor gets phosphorylated, which leads to the recruitment of dishevelled proteins and axin, thereby preventing phosphorylation of $\beta$-catenin. As a result, hypophosphorylated $\beta$-catenin accumulates in the cytoplasm, translocates to the nucleus, interacts with the T-cell specific transcription factor/ lymphoid enhancer-binding factor family of transcription factors, and regulates target gene expression. The reactivation of this pathway has been reported in several different diseases, mainly cancer [13]. Importantly, recent studies

\section{AFFILIATIONS}

*Dept of Medicine, University of Giessen Lung Center, University of Giessen, Giessen,

"Comprehensive Pneumology Center, Ludwig-MaximiliansUniversity, Asklepios Hospital, and "Helmholtz Zentrum München,

Munich, Germany.

CORRESPONDENCE

M. Königshoff

Comprehensive Pneumology Center, Ludwig Maximilians University Munich

University Hospital Grosshadern and Helmholtz Zentrum München Max-Labsche-Platz 31

81377 Munich Germany

E-mail: melanie.koenigshoff@ helmholtz-muenchen.de

Received:

Sept 082009

Accepted after revision: June 242010

First published online: July 222010 
have linked increased WNT/ $\beta$-catenin signalling to impaired epithelial function in the pathogenesis of IPF [4, 14-17].

The WNT/ $\beta$-catenin pathway is tightly controlled in a spatiotemporal manner. WNT regulators, such as proteins of the Dickkopf (DKK) family, are expressed in response to active WNT/ $\beta$-catenin signalling. Four different DKK proteins (DKK1-4) have been discovered, sharing conserved cysteinerich domains. DKK proteins bind to the LRP receptors and also bind to a second class of transmembrane receptors, called Kremen (KRM) [18], which potentiate the ability of DKK to regulate WNT signalling [19-21].

In this respect, we hypothesised that the WNT regulators DKK and KRM are differentially expressed in IPF, possibly affecting impaired epithelial injury and repair processes.

\section{MATERIAL AND METHODS}

\section{Human lung tissue}

Lung tissue biopsies were obtained from 15 IPF patients with histological UIP pattern (four females, 11 males: mean age $58 \pm 8$ yrs; mean vital capacity $48 \pm 7 \%$; mean total lung capacity $50 \pm 5 \%$; mean diffusing capacity of the lung for carbon monoxide per unit of alveolar volume $23 \pm 3 \%$; $\mathrm{O}_{2} 2$ $4 \mathrm{~L} \cdot \mathrm{min}^{-1}$; arterial oxygen tension 49-71 $\mathrm{mmHg}$; arterial carbon dioxide tension $33-65 \mathrm{mmHg}$ ) and nine control subjects (organ donors, four females, five males; mean age $42 \pm 10 \mathrm{yrs}$ ). Individual patient characteristics have been described previously [4]. Samples were immediately snap frozen or placed in $4 \%$ (weight/volume) paraformaldehyde after explantation. The study protocol was approved by the Ethics Committee of the Justus-Liebig-University School of Medicine, Giessen, Germany (AZ 31/93). Informed consent was obtained in written form from each subject for the study protocol.

\section{Human bronchial lavage fluids}

Patients were recruited at the Dept of Medicine at the JustusLiebig-University in 2006 and 2007. The study protocol was approved by the local ethics committee, and informed consent was obtained from the patients. Flexible fibreoptic bronchoscopy was performed in patients and controls by one physician in a standardised manner, as previously described [22]. Individual patient characteristics are shown in table 1. The control group consisted of four spontaneously breathing healthy nonsmoking volunteers, with normal pulmonary function, clinical blood tests without pathological findings, and without any history of cardiac or lung disease (medical students from the Medical School of the Justus-Liebig University).

\section{Reverse transcription and quantitative $R T-P C R$}

RNA extraction and quantitative (q)RT-PCR was performed using fluorogenic SYBR Green and the Sequence Detection System Fast 7500 (PE Applied Biosystems, Carlsbad, CA, USA), as previously described [4]. HPRT1, an ubiquitously and equally expressed gene free of pseudogenes, was used as a reference gene in all human qRT-PCR reactions. PCR primers are listed in table 2. Relative transcript abundance of a gene is expressed in $\Delta \mathrm{Ct}$ values:

$$
\Delta \mathrm{Ct}=\mathrm{Ct}^{\text {reference }}-\mathrm{Ct}^{\text {target }}
$$

Relative changes in transcript levels compared to donors are $\Delta \Delta \mathrm{Ct}$ values:

$$
\Delta \Delta \mathrm{Ct}=\Delta \mathrm{Ct} \mathrm{IPF}^{\mathrm{IP}}-\Delta \mathrm{Ct} \mathrm{tonor}^{\text {don }}
$$

All $\Delta \Delta \mathrm{Ct}$ values correspond approximately to the binary logarithm of the fold change (log-fold change) as mentioned in the article. When relative transcript abundance is given, expression levels are presented in $\Delta \mathrm{Ct}$ levels.

\section{Western blot analysis}

Human lung tissue was homogenised in extraction buffer and whole proteins were extracted by centrifugation $(12,000 \times g)$ for $10 \mathrm{~min}$ at $4^{\circ} \mathrm{C}$, as described previously [4]. The following antibodies were used: DKK1 (sc-25516; Santa Cruz Biotechnology, Santa Cruz, CA, USA), DKK2 and DKK4 (ab38594 and ab38589; Abcam, Cambridge, UK), KRM1 (AF2127; R\&D Systems, Minneapolis, MN, USA), and KRM2 (HP A003223; Sigma-Aldrich, Saint Louis, MO, USA). Densitometric analysis of autoradiographies was performed using a GS-800TM Calibrated Densitometer and the 1-D analysis software Quantity One (both from Bio-Rad Laboratories, Hercules, CA, USA). Changes in expression levels are expressed as fold change (mean \pm SEM).

\begin{tabular}{|c|c|c|c|c|c|c|c|c|c|}
\hline No. & Diagnosis & Sex & Age yrs & VC \% pred & DL,Co/VA \% pred & TLC \% pred & $\mathrm{O}_{2} \mathrm{~L} \cdot \mathrm{min}^{-1}$ & $\mathrm{~Pa}, \mathrm{O}_{2} \mathrm{mmHg}$ & $\mathrm{Pa}, \mathrm{CO}_{2} \mathrm{mmHg}$ \\
\hline 1 & IPF (UIP) & Male & 66 & 86 & 56 & 78 & 2 & 90 & 41 \\
\hline 2 & IPF (UIP) & Male & 76 & 41 & 73 & 47 & & 79 & 38 \\
\hline 3 & IPF (UIP) & Male & 68 & 57 & 37 & 55 & & 51 & 34 \\
\hline 4 & IPF (UIP) & Male & 60 & 33 & na & 42 & 5 & 69 & 41 \\
\hline 5 & IPF (UIP) & Male & 64 & 69 & 54 & 71 & & 70 & 35 \\
\hline 6 & IPF (UIP) & Male & 79 & 81 & 42 & 75 & & 45 & 37 \\
\hline 7 & IPF (UIP) & Male & 65 & 60 & 48 & 62 & & 61 & 34 \\
\hline 8 & IPF (UIP) & Male & 65 & 64 & 75 & 58 & & 78 & 35 \\
\hline 9 & IPF (UIP) & Male & 69 & 36 & NA & 41 & NA & 71 & 46 \\
\hline
\end{tabular}

\section{TABLE 1 Characteristics of idiopathic pulmonary fibrosis (IPF) patients}

VC: vital capacity; \% pred: \% predicted; $\mathrm{DL}, \mathrm{CO} / \mathrm{VA}$ : diffusing capacity of the lung for carbon monoxide per unit of alveolar volume; TLC: total lung capacity; $\mathrm{Pa}, \mathrm{O}_{2}$ : arterial oxygen tension; $\mathrm{Pa}_{2} \mathrm{CO}_{2}$ : arterial carbon dioxide tension; UIP: usual interstitial pneumonia; $\mathrm{NA}$ : not applicable. 
TABLE 2 Primer sequences and amplicon sizes for human tissues ${ }^{\#}$

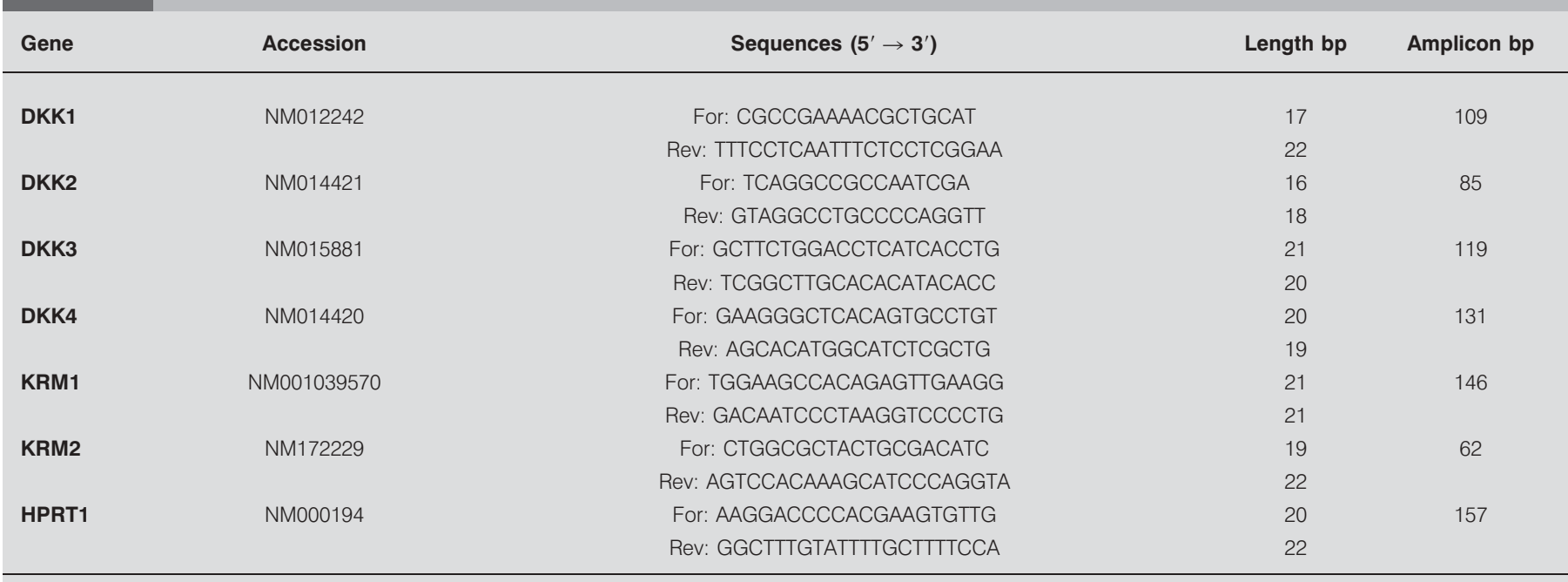

DKK: Dickkopf; KRM: Kremen; HPRT1: hypoxanthine guanine phosphoribosyl transferase 1; for: forward; rev: reverse. \#: All primer sets worked under identical real-time PCR cycling conditions with similar efficiencies to obtain simultaneous amplification in the same run. Sequences were taken from GeneBank, all accession numbers are denoted.
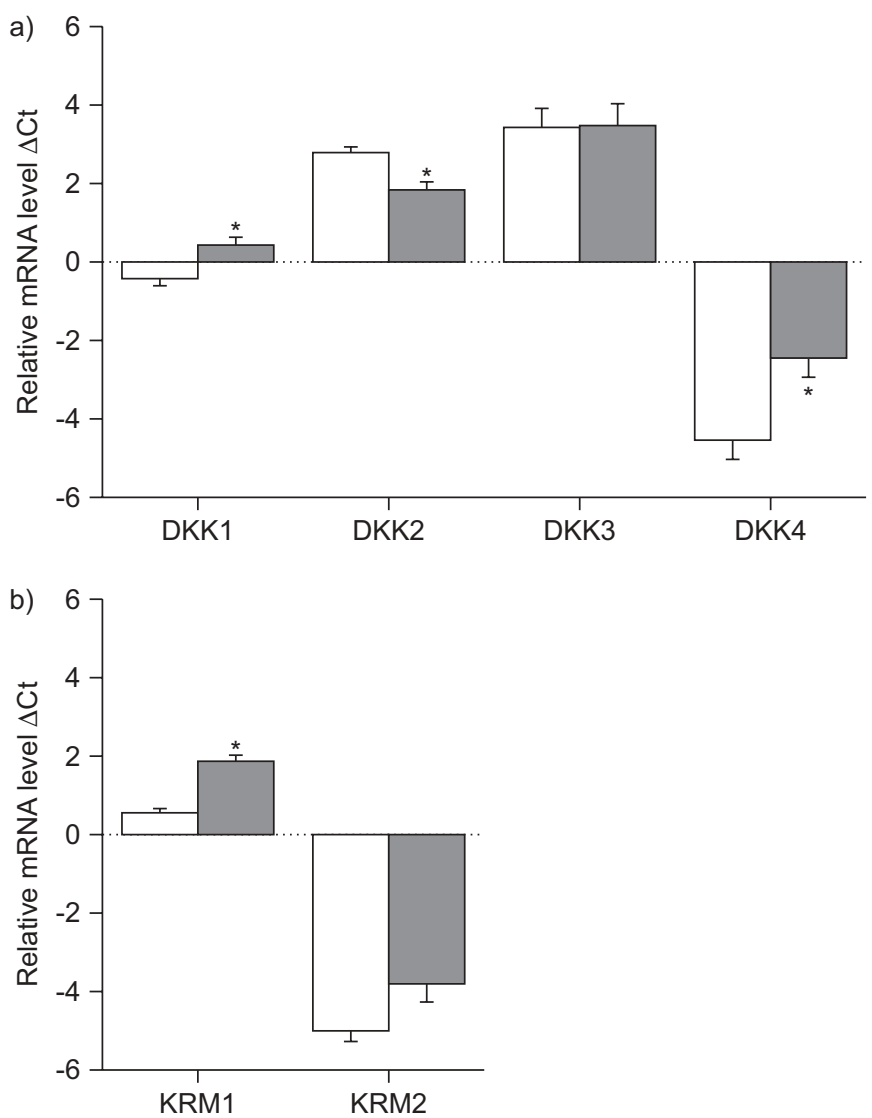

FIGURE 1. The mRNA expression of Dickkopf (DKK) and Kremen (KRM) proteins in idiopathic pulmonary fibrosis (IPF) and donor tissue. The mRNA expression of a) DKK1-4 and b) the receptors KRM1 and 2 was analysed in total lung homogenates from donor $(\square)$ and IPF lung specimen ( $\square$ ) by quantitative RT-PCR Results from 10 donors and 10 IPF patients are shown as relative mRNA expression compared to the reference gene $(\Delta \mathrm{Ct})$, and presented as mean \pm SEM. $*$ : $p<0.05$.

\section{Immunohistochemistry}

Human lungs were placed in $4 \%(\mathrm{w} / \mathrm{v})$ paraformaldehyde after explantation, and processed for paraffin embedding. Sections $(3 \mu \mathrm{m})$ were cut, mounted on slides, and subjected to antigen retrieval and quenching of endogenous peroxidase activity using 3\% (volume/volume) hydrogen peroxide for $20 \mathrm{~min}$. The following antibodies were used: DKK1 and DKK4 (sc-25516 and sc-25519; Santa Cruz Biotechnology), KRM1 (AF2127; R\&D Systems), KRM2 (HP A003223, Sigma-Aldrich). Immune complexes were visualised using peroxidase-coupled secondary antibodies, according to the manufacturer's protocol (Histostain Plus Kit; Invitrogen, Camarillo, CA, USA).

\section{ELISA}

A human DKK1 ELISA (DY1906; R\&D Systems) was performed on bronchoalveolar lavage fluid (BALF) following the manufacturers' instructions. $100 \mu \mathrm{L}$ of BALF was used, each sample was measured twice. A seven point standard curve with a high standard of $4,000 \mathrm{pg} \cdot \mathrm{mL}^{-1}$ and two-fold serial dilutions to a low standard $\left(75 \mathrm{pg} \cdot \mathrm{mL}^{-1}\right)$ was performed at the same plate, two measurements for each dilution. Double values of samples and standards were averaged.

\section{Cell culture}

The human bronchial epithelial cell line BEAS-2B (European Collection of Cell Cultures) was maintained in LHC-9 medium (Invitrogen). The human lung epithelial cell line A549 (ATCC CCL-185; American Type Culture Collection, Manassas, VA, USA) was maintained in Dulbecco's modified Eagle medium (GIBCO; Invitrogen), supplemented with $10 \%$ fetal bovine serum (PAA Laboratories, Pasching, Austria). Cells were plated in 24-well plates, serum starved for $20 \mathrm{~h}$ in $0.1 \%$ fetal calf serum medium. Stimulation for $24 \mathrm{~h}$ was performed with recombinant WNT3a or DKK1 (both from R\&D Systems), as indicated. Cell counting was performed using a haemacytometer according to standard protocols. 


\section{Statistical analysis}

All $\Delta$ Ct values obtained from real-time RT-PCR were analysed for normal distribution using the Shapiro-Wilk test, using assignment of a normal distribution with $\mathrm{p}>0.05$. Normality of data was confirmed using quantile-quantile plots. The means of indicated groups were compared using a two-tailed unpaired t-test, or a ANOVA with Tukey HSD post hoc test for studies with more than two groups. Results were considered statistically significant when $\mathrm{p}<0.05$.

\section{RESULTS}

Initially, we quantified the mRNA expression of DKK1-4 and KRM1 and 2 in homogenised lung tissue specimens of transplant donors and IPF patients $(n=10$ each) using (q)RTPCR. As demonstrated in figure $1 \mathrm{a}$, all DKK proteins were expressed in donor and IPF lungs, but exhibited variable basal expression levels. DKK3 was highly expressed in both donor and IPF tissue, whereas DKK4 showed the lowest mRNA level in both conditions. DKK1 and DKK4 presented significantly increased mRNA expression in fibrotic tissue (log-fold change (mean \pm SEM): $0.85 \pm 0.29$ and $2.09 \pm 0.70$, respectively), while DKK2 mRNA was decreased (log-fold change: $-0.94 \pm 0.27$ ). The receptors KRM1 and KRM2 were also expressed in lung tissue, with lower expression of KRM2 compared with KRM1 in donor and IPF tissue. In IPF, enhanced KRM1 mRNA levels were detected (log-fold change: $1.31 \pm 0.21$ ) (fig. $1 b$ ).

a)

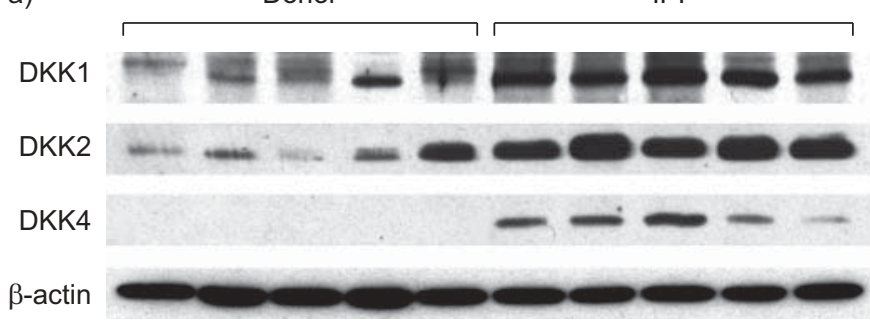

Next, we analysed the protein expression pattern of DKK and KRM proteins in lung homogenates of donors and IPF patients $(n=5$ each). As depicted in figure 2, Western blotting of DKK proteins showed enhanced levels of all investigated DKK proteins in IPF. Quantification of immunoblots demonstrated significantly increased intensity for DKK1 and DKK4 (fig. 2a and $b$ ) (increases in optical density versus $\beta$-actin, DKK1 $0.24 \pm 0.08$ and DKK4 $0.62 \pm 0.08$ ), which is in accordance to elevated transcript levels depicted in figure 1. Protein expression of DKK2 was also significantly enhanced $(0.52 \pm 0.1)$ in IPF compared with lung tissue from transplant donors, however, transcript levels were decreased in IPF lung specimens (fig. 1a). Both receptors KRM1 and 2 were expressed in the lung (fig. 2c), with significantly increased expression of KRM1 $(0.45 \pm 0.04)$ (fig. $2 \mathrm{~d})$

We then sought to identify the cells capable of expressing DKK ligands and KRM receptors. Therefore, we performed immunohistochemical stainings on IPF and donor lung sections. As demonstrated in figure 3, DKK1 was mainly localised in bronchial epithelial cells in donor and IPF lungs (fig. 3a). Interestingly, we observed a pronounced and distinct accumulation of DKK1 in basal bronchial epithelial cells (fig. 3a, arrows). In IPF lungs, DKK1 was particularly localised in hyperplastic alveolar epithelial cells (fig. $3 \mathrm{~b}$, arrows). In addition, granulocytes (fig. 3b) presented staining of DKK1 protein. DKK4 protein expression was largely localised to bronchial

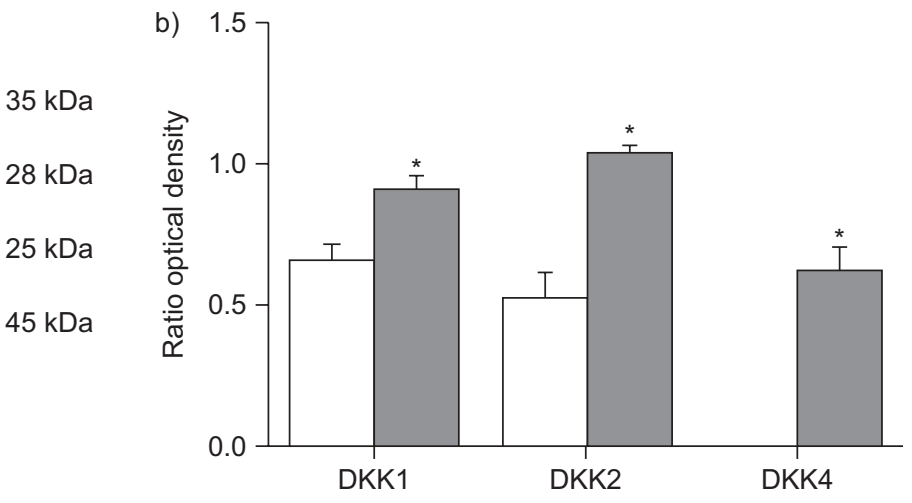

c)

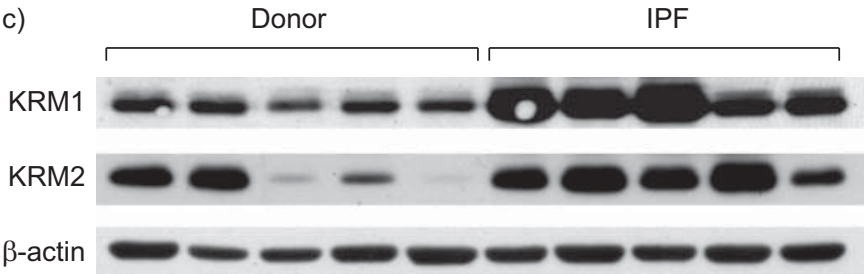

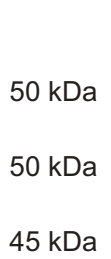

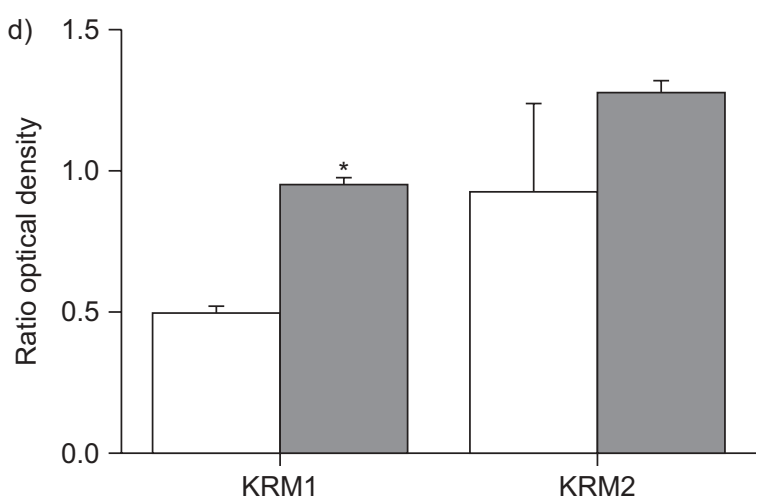

FIGURE 2. Protein expression of Dickkopf (DKK) and Kremen (KRM) in lung homogenates of donors ( $\square$ ) and idiopathic pulmonary fibrosis (IPF; $\square$ ) patients. a, b) Expression of DKK1, 2 and 4 and c, d) KRM1 and 2 in total protein lysates of donor and IPF lung homogenates was determined by Western blot analysis. Antibodies were used as indicated, $\beta$-actin served as a loading control. Immunoblots were carried out at least twice, a representative blot is shown (a and c). Densitometry is shown in b and d, respectively. Ratio of optical density (optical density of indicated protein/optical density of $\beta$-actin) is presented for donor and IPF tissues as mean \pm SEM. The ratio optical density in donors for DKK4 was not available. *: $p<0.05$. 
a)
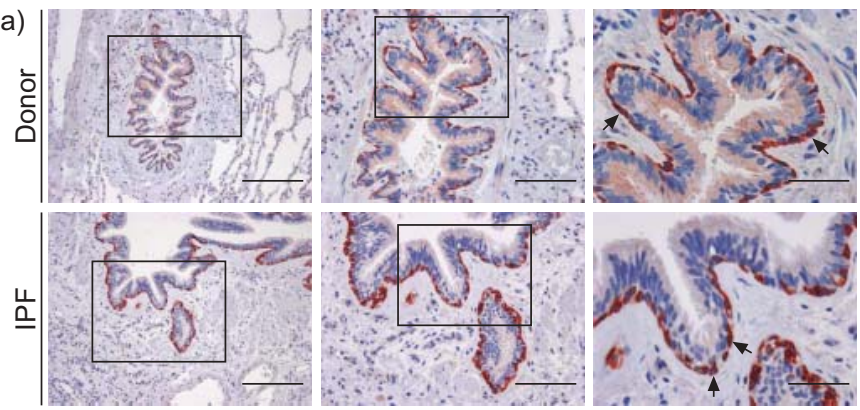

b)
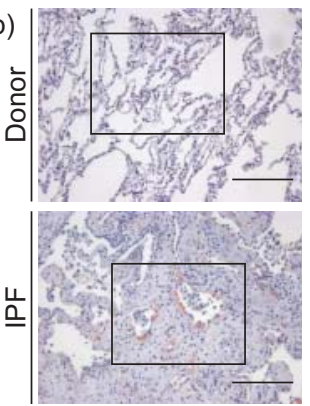
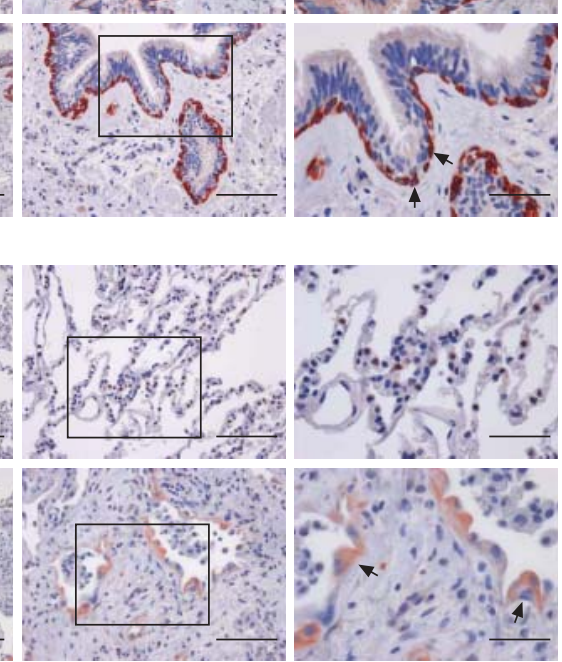

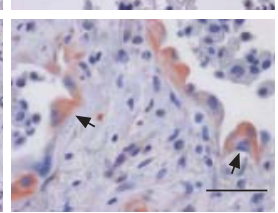

FIGURE 3. Expression and localisation of Dickkopf (DKK)1 in lung tissue of donors and idiopathic pulmonary fibrosis (IPF) patients. Immunohistochemistry for DKK1 was performed at lung tissue sections of donors and IPF patients. Stainings were performed at least twice using three different donor and IPF lungs. Representative a) bronchiolar and b) alveolar regions for donor and IPF lungs are shown in three magnifications (200, 100 and $50 \mu \mathrm{m}$ from left to right). Arrows indicate basal bronchial epithelial cells (a) and hyperplastic alveolar epithelial cells (b)

epithelial cells and interstitial cells, in donor as well as IPF tissues. Of note, DKK4 expression exhibited an equal basalapical intensity in bronchial epithelial cells (fig. 4a) in donor and IPF tissue. As depicted in figure 4b, DKK4 was strongly expressed in hyperplastic alveolar epithelial cells and areas of bronchiolisation in IPF (fig. 4b, arrows).

We went on to localise the expression of the DKK-binding receptors KRM1 and KRM2. KRM1 protein exhibited expression in bronchial epithelial (fig. 5a), smooth muscle cells (fig. 5a, arrows) and endothelial cells (fig. 5a) in donor lung tissue. In IPF, a heterogeneous staining of the bronchial epithelium (fig. 5a) and in hyperplastic alveolar cell regions was dominant (fig. 5b, arrows). KRM1 was also detected in alveolar macrophages in donor lung tissue (fig. $5 \mathrm{a}$ and b). Similarly, scattered protein expression of KRM2 was localised to bronchial epithelial cells (fig. 6a) in donor and IPF lungs. In IPF, hyperplastic alveolar epithelial cells expressed KRM2 (fig. 6b, arrow).

Taken together, these results demonstrated increased expression of the DKK ligands and their receptors in IPF. All proteins largely localised to the lung epithelium, suggesting that epithelial cells respond to secreted DKK ligands in an autocrine fashion. To further elucidate this, we next determined the DKK1 concentration in the bronchial lumen. DKK1 protein was quantified in BALF of healthy volunteers $(n=4)$, patients with chronic bronchitis $(n=3)$ or IPF $(n=9)$ using an
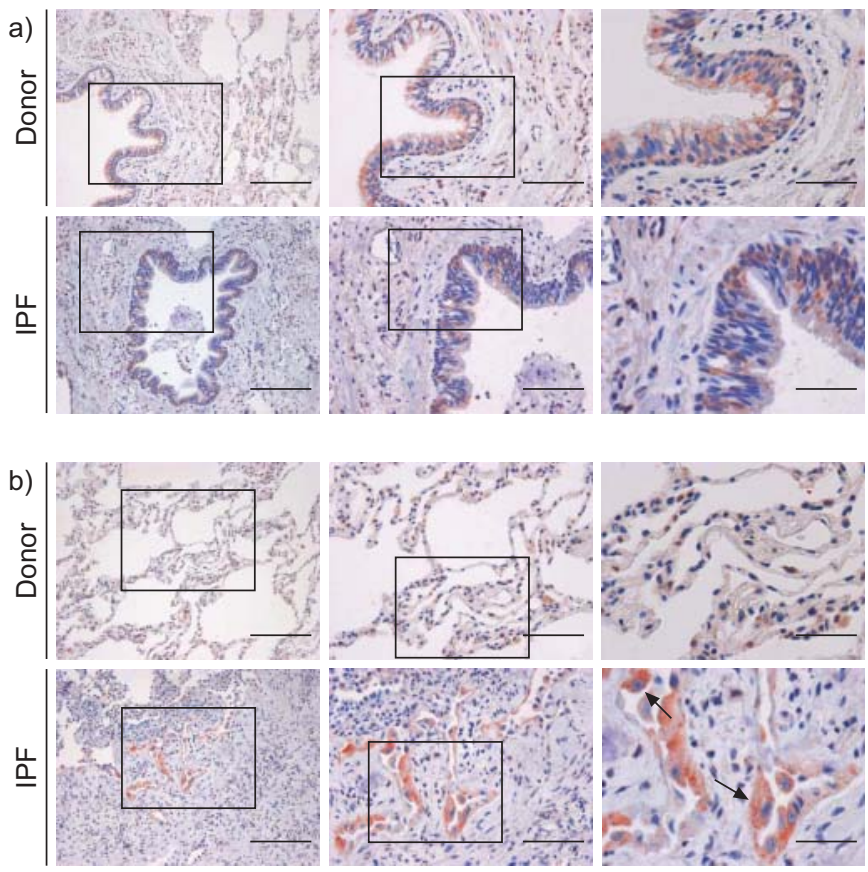

FIGURE 4. Expression and localisation of Dickkopf (DKK)4 in lung tissue of donors and idiopathic pulmonary fibrosis (IPF) patients. Immunohistochemistry for DKK4 was performed on lung tissue sections of donor and IPF patients. Stainings were performed at least twice using three different donor and IPF lungs. Representative a) bronchiolar and b) alveolar regions for donor and IPF lungs are shown in three magnifications (200, 100 and $50 \mu \mathrm{m}$ from left to right). Arrows indicate positive hyperplastic alveolar epithelial cells.

ELISA. As depicted in figure 7, DKK1 was expressed in all investigated samples, with a significantly increased amount of DKK1 in BALF of IPF patients (mean \pm SEM $456 \pm 44 \mathrm{pg} \cdot \mathrm{mL}^{-1}$ ), compared with healthy controls $\left(266 \pm 8 \mathrm{pg} \cdot \mathrm{mL}^{-1}\right)$ or patients with chronic bronchitis $\left(223 \pm 34 \mathrm{pg} \cdot \mathrm{mL}^{-1}\right)$.

Finally, we wanted to explore the effects of DKK1 on lung epithelial cell function. To this end, we stimulated the human bronchial epithelial cell line BEAS-2B or the human lung epithelial cell line A549 with recombinant WNT3a, DKK1 or a combination thereof, and analysed the effects on epithelial cell proliferation. As presented in figure 8a, stimulation of BEAS2B with WNT3a induced a significant increase in cell proliferation compared with controls (relative proliferation (mean \pm SEM) $1.39 \pm 0.06$ ). Interestingly, low concentrations of DKK1 (100 $\left.\mathrm{ng} \cdot \mathrm{mL}^{-1}\right)$ alone also led to a significant increase in bronchial epithelial cell proliferation and failed to inhibit WNT3a-induced effects significantly $(1.6 \pm 0.14$ versus $1.41 \pm$ 0.13 , respectively). Higher concentrations of DKK1 led to a reduction of WNT3a-induced effects, while no significant effect on cell proliferation was observed after stimulation with DKK1 alone. Similar to bronchial epithelial cells, WNT3a increased the proliferative capacity of alveolar epithelial cells (fig. 8b) (relative proliferation (mean \pm SEM) $1.42 \pm 0.08$ ), which was attenuated by high concentrations of DKK1 (DKK1 $500 \mathrm{ng} \cdot \mathrm{mL}^{-1} 1.07 \pm 0.05$, and DKK1 $\left.1,000 \mathrm{ng} \cdot \mathrm{mL}^{-1} 1.11 \pm 0.08\right)$. The effect of lower concentration of DKK1, however, was different, as DKK1 treatment alone did not lead to an increase in alveolar epithelial cells proliferation. 

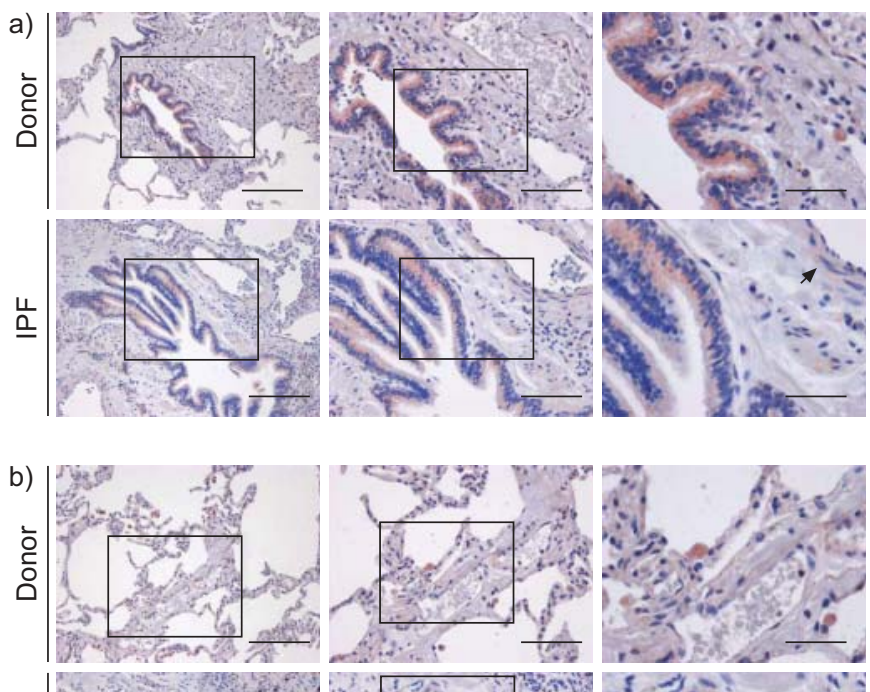

$\underline{\underline{\underline{a}}}$
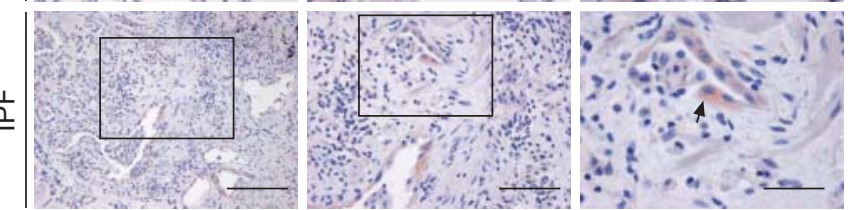

FIGURE 5. Expression and localisation of Kremen (KRM)1 in lung tissue of donors and idiopathic pulmonary fibrosis (IPF) patients. Immunohistochemistry for KRM1 was performed on lung tissue sections of donor and IPF patients. Stainings were performed at least twice using three different donor and IPF lungs. Representative a) bronchiolar and b) alveolar regions for donor and IPF lungs are shown in three magnifications (200, 100 and $50 \mu \mathrm{m}$ from left to right). Arrows indicate positive smooth muscle cells (a) and hyperplastic alveolar epithelium (b).

\section{DISCUSSION}

IPF is a progressive and fatal lung disease with limited responsiveness to current therapies [2, 3]. The molecular mechanisms involved in IPF are still poorly understood. The WNT/ $\beta$-catenin pathway, known to be critical during lung morphogenesis and associated with the development of lung carcinoma [13, 23], has recently been demonstrated to be expressed and active in IPF, modulating epithelial cell injury and repair $[4,14,15]$. Herein, we performed a comprehensive analysis of the expression and localisation of the WNT modulators DKK and their KRM receptors, demonstrating that both DKK and KRM proteins are enhanced in lung tissue specimens of donors and IPF patients. Importantly, the expression analysis was performed in lung homogenate samples, which implies that the expression profiles are subject to the cellular composition of the samples used. Immunohistochemical analysis revealed that DKK and KRM proteins largely localise to lung epithelial cells. Of note, DKK1 exhibited strong expression in basal bronchial and hyperplastic alveolar epithelial cells in IPF. Analysis of BALF revealed increased DKK1 expression in the bronchoalveolar lumen in IPF. Furthermore, in vitro studies demonstrated that DKK1 alter WNT-induced epithelial cell proliferation in a dosedependent fashion.

The WNT signalling system is tightly controlled by different secreted WNT regulators, such as the secreted frizzled receptor (sFRP) or DKK proteins [21, 24]. Both protein families use a fundamentally different mechanism to modulate WNT signalling.
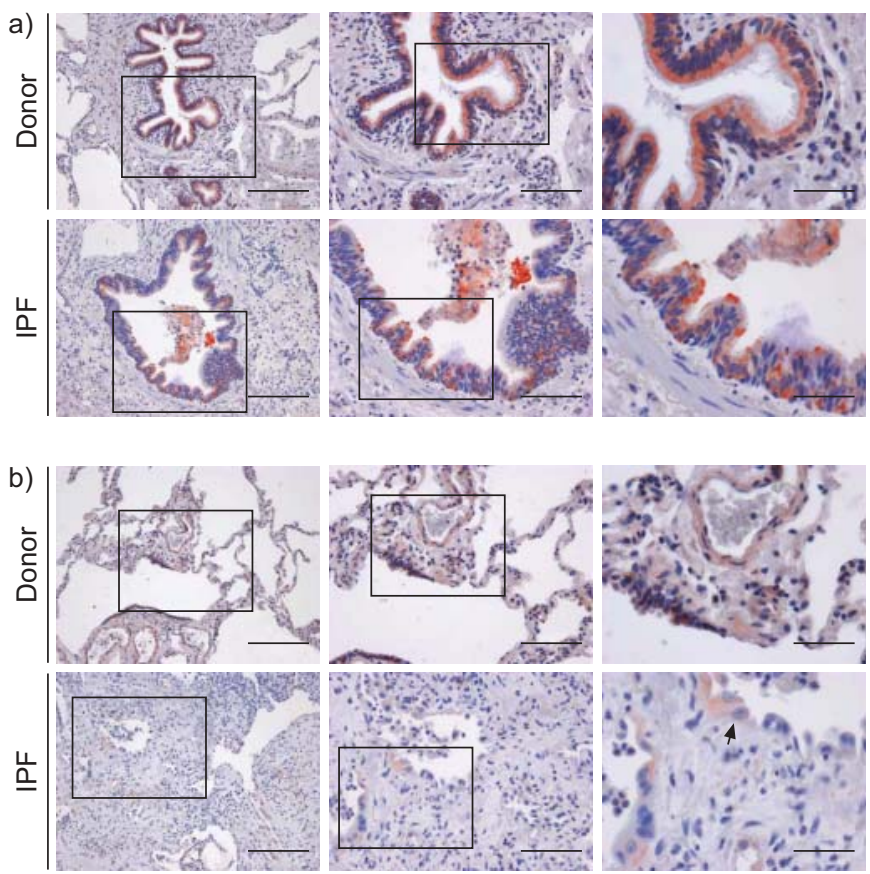

FIGURE 6. Expression and localisation of Kremen (KRM)2 in lung tissue of donor and idiopathic pulmonary fibrosis (IPF) patients. Immunohistochemistry for KRM2 was performed on lung tissue sections of donor and IPF patients. Stainings were performed at least twice using three different donor and IPF lungs. Representative a) bronchiolar and b) alveolar regions for donor and IPF lungs are shown in three magnifications (200, 100 and $50 \mu \mathrm{m}$ from left to right). Arrows indicate hyperplastic alveolar epithelial cells.

While sFRP bind directly to WNT ligands and inhibit their interaction with the membrane receptors frizzled or low-density LRP, DKK modulate the WNT/ $\beta$-catenin pathway by binding directly to LRP receptors and KRM receptors. The formation of a ternary complex of DKK, KRM and LRP6 is thought to lead to the internalisation of the whole complex from the cell surface, thereby inhibiting WNT signalling [21].

The potential of sFRP to modulate organ fibrosis has been demonstrated in the kidney in vivo and in vitro [25, 26], and sFRPs have been reported to be differentially expressed in pulmonary fibrosis [15]. With respect to the DKK family, most studies to date have focused on DKK1. Inhibition of WNT/ $\beta$ catenin signalling by DKK1 has been demonstrated in mouse lung organ cultures in vivo [27], however, the potential of DKK1 to modulate a fibrotic response via inhibition of WNT signalling has only been demonstrated in hepatic stellate cells [28], as well as in irradiated fibroblasts in vitro [29]. Recently, DKK1 has also been implicated in the development of rheumatoid arthritis [30]. Herein, we report for the first time that proteins from the DKK family are differentially regulated in IPF.

DKK1 is the founding member of the DKK family and originally identified as embryonic head inducer and WNT inhibitor in Xenopus [21]. In contrast, DKK2 has been described to act as a WNT antagonist as well as a WNT agonist, depending on the cellular context and the availability of WNTand co-receptors [21]. In addition, DKK1 is known to be a direct target gene upregulated after WNT stimulation [31], whereas for DKK2 this has not been demonstrated yet. Our 


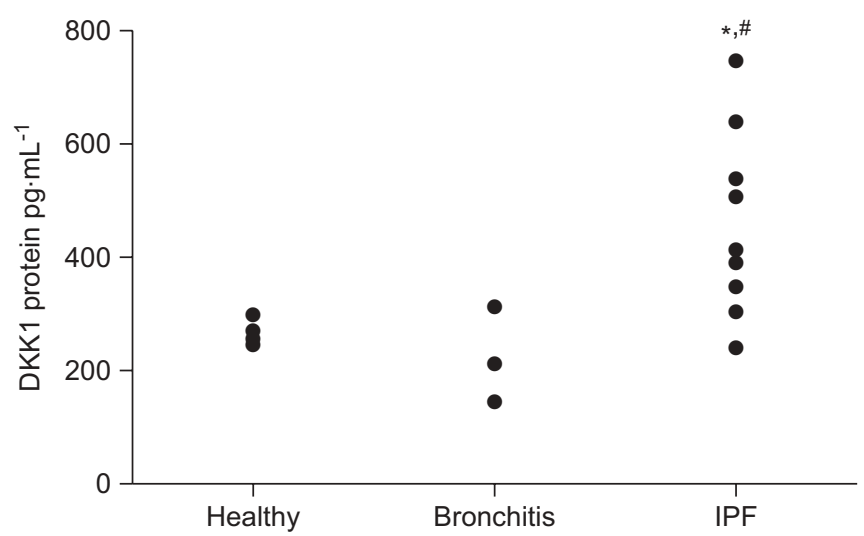

FIGURE 7. Quantification of Dickkopf (DKK) 1 in bronchoalveolar lavage fluid (BALF) from healthy volunteers, patients with chronic bronchitis and idiopathic pulmonary fibrosis (IPF) patients. DKK1 protein concentration in BALF of healthy volunteers, patients with chronic bronchitis and IPF patients was quantified using an ELISA. Results are derived from four healthy volunteers, three patients with chronic bronchitis and nine IPF patients. *: $p<0.05$ compared with healthy volunteers; $\#$ : $p<0.05$ compared with chronic bronchitis.

study also suggests that the transcriptional (feedback) control or protein stability due to post-translational processing may differ between DKK proteins.

The availability of DKK receptors in the lung is a basic requirement for secreted DKK proteins to exert their effects on $\mathrm{WNT} / \beta$-catenin signalling. Expression of LRP5 and 6 in lung homogenates of donors and IPF patients has been demonstrated in a recent study [4]. We focussed on the receptors KRM1 and 2. Immunohistochemical staining of KRM1 and 2 revealed that the bronchial and hyperplastic alveolar epithelium, in particular in areas of bronchiolisation of IPF specimen, are major sources in donor and IPF lung tissue specimens, indicating autocrine effects on epithelial cells as the main signalling mechanism for the WNT/ $\beta$-catenin pathway. The receptors KRM1 and 2, however, demonstrated a heterogeneous expression pattern in the epithelium, which highlights the importance of the microenvironment influencing WNT signalling in vivo. In addition, it has to be pointed out that fibroblasts have been recently reported to be capable of WNT signal transduction $[4,32]$, and also take part in the fibrotic process induced by WNT/ $\beta$-catenin signalling.

Importantly, DKK1 concentrations were only increased in BALF of IPF patients compared with healthy volunteers, but not in patients with chronic bronchitis, suggesting that WNT/ $\beta$-catenin activation and regulation does not primarily reflect an advanced inflammatory response.

Notably, we observed a distinct expression pattern for DKK1 with strong staining in basal bronchial epithelial cells in donor as well as in IPF lungs. Basal cells exhibit a proliferative activity and are thought to be important progenitors for the maintenance of the bronchial epithelium in general, and after lung injury in particular $[33,34]$. In addition, basal bronchial epithelial cells are also known to be involved in the development of lung cancer. Squamous cell carcinoma accounts for $20 \%$ of all human lung cancers and basal cell metaplasia is a premalignant finding in the bronchial
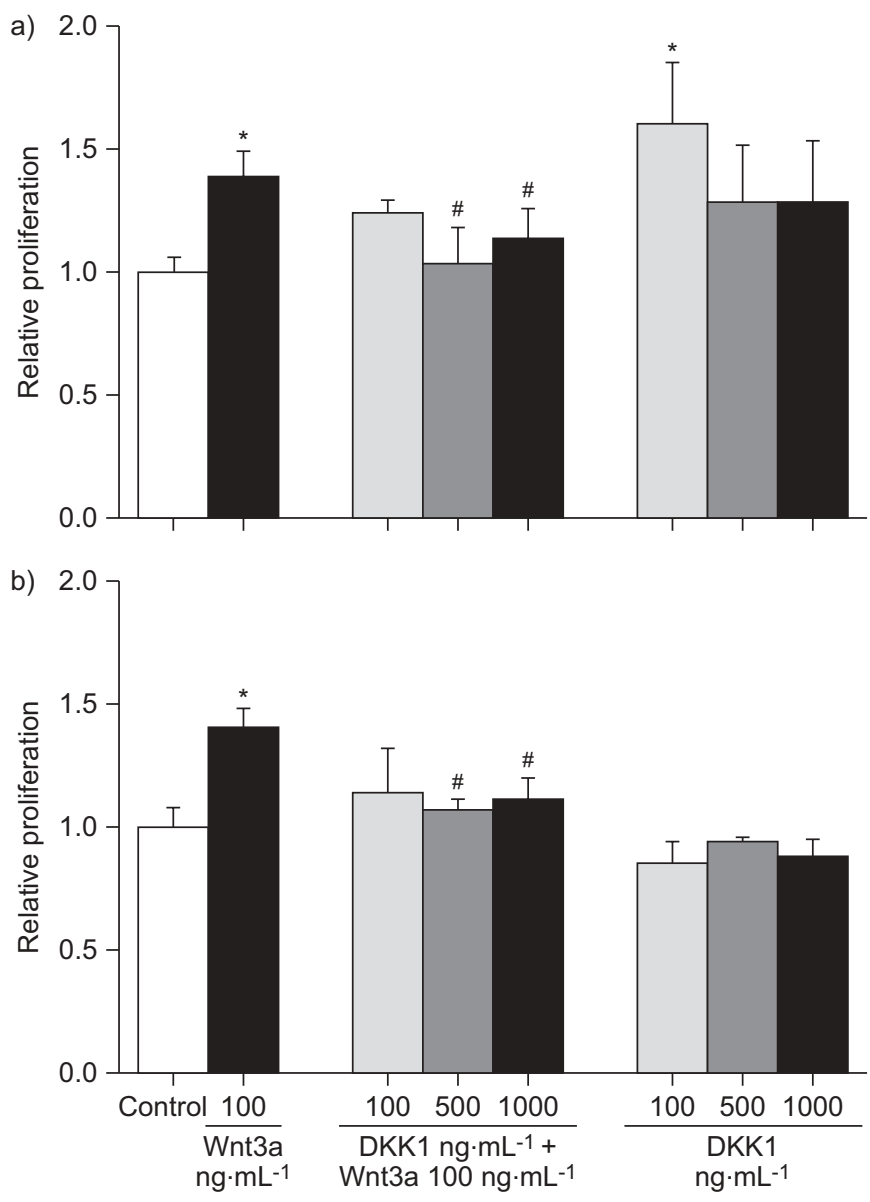

FIGURE 8. Effects of Dickkopf (DKK)1 and WNT3a on epithelial cell proliferation. a) BEAS-2B cells or b) A549 cells were stimulated with WNT3a, DKK1 or a combination thereof, as indicated. Proliferation was assessed by cell counting using a haemacytometer according to standard protocols. Data is shown as relative counts compared with control. Results are derived from four independent experiments and presented as mean \pm SEM. *: $p<0.05$ compared with control; \# : $p<0.05$ compared with WNT3a stimulated cells.

epithelium [35]. Importantly, bronchial epithelial cell metaplasia is also a common feature in IPF lung tissue specimens [36]. In addition, CHILOSI et al. [37] reported abnormal proliferation of bronchial epithelial cells in IPF, but not other interstitial pneumonias, such as acute or nonspecific pneumonias. It has been suggested that patients with bronchial epithelial cell metaplasia tend to develop lung carcinomas [38]. The WNT/ $\beta$ catenin pathway has been implicated in epithelial proliferation and it has been demonstrated that primary bronchial epithelial cells exhibit the potential to respond to WNT signalling [39].

We analysed the proliferative capacity of bronchial and alveolar epithelial cells revealing that only high concentrations of DKK1 inhibited the WNT-induced proliferative effect. Notably, low concentrations of DKK1 alone led to increased bronchial cell proliferation, but not alveolar epithelial cell proliferation. These results allow the assumption that DKK proteins modulate bronchial epithelial cell maintenance, and may be involved in an increased bronchial cell metaplasia, possibly leading to increased lung cancer development. Furthermore, our data suggest that DKK1, although expressed 
and secreted by the alveolar epithelium, is not able to fulfil an effective negative feedback-loop on WNT-induced aberrant alveolar epithelial cell proliferation in IPF in vivo. The effects of DKK on WNT signalling crucially depend on the concentration of the respective WNT or DKK ligands, as well as on the sensitivity of the effector cells due to receptor availability. It has to be pointed out, however, that the mere use of cell lines is a significant limitation of the current study, the results of which need to be analysed in more detail using primary alveolar and bronchial epithelial cells in future studies.

In summary, our study demonstrated altered expression of the WNT regulators DKK and KRM, which may be crucial for lung epithelial cell injury and repair mechanisms in IPF. Further studies are needed to elucidate the effects of DKK proteins on different cell types to reveal the potential therapeutic capability in IPF.

\section{SUPPORT STATEMENT}

The Clinical Research Group 118 "Lung Fibrosis", European 6th Framework Programme (PULMOTENSION), and the Career Development Grant ("Anschubfinanzierung") from the School of Medicine, Justus-Liebig-University Giessen, Germany funded this study.

\section{STATEMENT OF INTEREST}

None declared.

\section{ACKNOWLEDGEMENTS}

The authors would like to thank W. Klepetko (Dept of Cardiothoracic Surgery, University of Vienna, Vienna, Austria) and R. Voswinckel (Dept of Medicine, University of Giessen Lung Center, University of Giessen, Giessen, Germany) for providing human lung tissues and S. Heinemann (Dept of Medicine, University of Giessen Lung Center) for excellent help with patient sampling.

\section{REFERENCES}

1 American Thoracic Society/European Respiratory Society international multidisciplinary consensus classification of the idiopathic interstitial pneumonias, Am J Respir Crit Care Med 2002; 165: 277-304.

2 Martinez FJ, Safrin S, Weycker D, et al. The clinical course of patients with idiopathic pulmonary fibrosis. Ann Intern Med 2005; 142: 963-967.

3 Walter N, Collard HR, King TE Jr. Current perspectives on the treatment of idiopathic pulmonary fibrosis. Proc Am Thorac Soc 2006; 3: 330-338.

4 Konigshoff M, Balsara N, Pfaff EM, et al. Functional Wnt signaling is increased in idiopathic pulmonary fibrosis. PLoS One 2008; 3: e2142.

5 Kim DS, Collard HR, King TE Jr. Classification and natural history of the idiopathic interstitial pneumonias. Proc Am Thorac Soc 2006; 3: 285-292.

6 Katzenstein AL, Myers JL. Idiopathic pulmonary fibrosis: clinical relevance of pathologic classification. Am J Respir Crit Care Med 1998; 157: 1301-1315.

7 Visscher DW, Myers JL. Histologic spectrum of idiopathic interstitial pneumonias. Proc Am Thorac Soc 2006; 3: 322-329.

8 Horowitz JC, Thannickal VJ. Epithelial-mesenchymal interactions in pulmonary fibrosis. Semin Respir Crit Care Med 2006; 27: 600-612.

9 Selman M, King TE, Pardo A. Idiopathic pulmonary fibrosis: prevailing and evolving hypotheses about its pathogenesis and implications for therapy. Ann Intern Med 2001; 134: 136-151.
10 Noble PW, Homer RJ. Back to the future: historical perspective on the pathogenesis of idiopathic pulmonary fibrosis. Am J Respir Cell Mol Biol 2005; 33: 113-120.

11 White ES, Lazar MH, Thannickal VJ. Pathogenetic mechanisms in usual interstitial pneumonia/idiopathic pulmonary fibrosis. J Pathol 2003; 201: 343-354.

12 Logan CY, Nusse R. The Wnt signaling pathway in development and disease. Annu Rev Cell Dev Biol 2004; 20: 781-810.

13 Moon RT, Kohn AD, De Ferrari GV, et al. WNT and betacatenin signalling: diseases and therapies. Nat Rev Genet 2004; 5: 691-701.

14 Chilosi M, Poletti V, Zamo A, et al. Aberrant Wnt/beta-catenin pathway activation in idiopathic pulmonary fibrosis. Am J Pathol 2003; 162: 1495-1502.

15 Selman M, Pardo A, Kaminski N. Idiopathic pulmonary fibrosis: aberrant recapitulation of developmental programs? PLoS Med 2008; 5: e62.

16 Konigshoff M, Eickelberg O. WNT signaling in lung disease: a failure or a regeneration signal? Am J Respir Cell Mol Biol 2010; 42: 21-31.

17 Konigshoff M, Kramer M, Balsara N, et al. WNT1-inducible signaling protein-1 mediates pulmonary fibrosis in mice and is upregulated in humans with idiopathic pulmonary fibrosis. J Clin Invest 2009; 119: 772-787.

18 Mao B, Niehrs C. Kremen2 modulates Dickkopf2 activity during Wnt/LRP6 signaling. Gene 2003; 302: 179-183.

19 Nakamura T, Nakamura T, Matsumoto K. The functions and possible significance of Kremen as the gatekeeper of Wnt signalling in development and pathology. J Cell Mol Med 2008; 12: 391-408.

20 Wang $\mathrm{K}$, Zhang $\mathrm{Y}, \mathrm{Li}$ X, et al. Characterization of the Kremenbinding site on Dkk1 and elucidation of the role of Kremen in Dkkmediated Wnt antagonism. J Biol Chem 2008; 283: 23371-23375.

21 Niehrs C. Function and biological roles of the Dickkopf family of Wnt modulators. Oncogene 2006; 25: 7469-7481.

22 Markart P, Luboeinski T, Korfei M, et al. Alveolar oxidative stress is associated with elevated levels of non-enzymatic low-molecularweight antioxidants in patients with different forms of chronic fibrosing interstitial lung diseases. Antioxid Redox Signal 2008; 11: 227-240.

23 Van Scoyk M, Randall J, Sergew A, et al. Wnt signaling pathway and lung disease. Transl Res 2008; 151: 175-180.

24 Kawano Y, Kypta R. Secreted antagonists of the Wnt signalling pathway. J Cell Sci 2003; 116: 2627-2634.

25 Hermens JS, Thelen P, Ringert RH, et al. Alterations of selected genes of the Wnt signal chain in rat kidneys with spontaneous congenital obstructive uropathy. J Pediatr Urol 2007; 3: 86-95.

26 Surendran K, Schiavi S, Hruska KA. Wnt-dependent beta-catenin signaling is activated after unilateral ureteral obstruction, and recombinant secreted frizzled-related protein 4 alters the progression of renal fibrosis. J Am Soc Nephrol 2005; 16: 2373-2384.

27 De Langhe SP, Sala FG, Del Moral PM, et al. Dickkopf-1 (DKK1) reveals that fibronectin is a major target of Wnt signaling in branching morphogenesis of the mouse embryonic lung. Dev Biol 2005; 277: 316-331.

28 Cheng JH, She H, Han YP, et al. Wnt antagonism inhibits hepatic stellate cell activation and liver fibrosis. Am J Physiol Gastrointest Liver Physiol 2008; 294: G39-G49.

29 Gurung A, Uddin F, Hill RP, et al. $\beta$-catenin is a mediator of the response of fibroblasts to irradiation. Am J Pathol 2009; 174: 248-255.

30 Diarra D, Stolina M, Polzer $\mathrm{K}$, et al. Dickkopf-1 is a master regulator of joint remodeling. Nat Med 2007; 13: 156-163.

31 Niida A, Hiroko T, Kasai M, et al. DKK1, a negative regulator of Wnt signaling, is a target of the beta-catenin/TCF pathway. Oncogene 2004; 23: 8520-8526. 
32 Vuga LJ, Ben-Yehudah A, Kovkarova-Naumovski E, et al. WNT5A is a regulator of fibroblast proliferation and resistance to apoptosis. Am J Respir Cell Mol Biol 2009; 41: 583-589.

33 Haij R, Baranek T, Le Naour R, et al. Basal cells of the human adult airway surface epithelium retain transit-amplifying cell properties. Stem Cells 2007; 25: 139-148.

34 Hong KU, Reynolds SD, Watkins S, et al. Basal cells are a multipotent progenitor capable of renewing the bronchial epithelium. Am J Pathol 2004; 164: 577-588.

35 Meyer EC, Liebow AA. Relationship of interstitial pneumonia honeycombing and atypical epithelial proliferation to cancer of the lung. Cancer 1965; 18: 322-351.
36 Hironaka M, Fukayama M. Pulmonary fibrosis and lung carcinoma: a comparative study of metaplastic epithelia in honeycombed areas of usual interstitial pneumonia with or without lung carcinoma. Pathol Int 1999; 49: 1060-1066.

37 Chilosi M, Poletti V, Murer B, et al. Abnormal re-epithelialization and lung remodeling in idiopathic pulmonary fibrosis: the role of deltaN-p63. Lab Invest 2002; 82: 1335-1345.

38 Aubry MC, Myers JL, Douglas WW, et al. Primary pulmonary carcinoma in patients with idiopathic pulmonary fibrosis. Mayo Clin Proc 2002; 77: 763-770.

39 Steel MD, Puddicombe SM, Hamilton LM, et al. Beta-catenin/T-cell factor-mediated transcription is modulated by cell density in human bronchial epithelial cells. Int J Biochem Cell Biol 2005; 37: 1281-1295. 